\title{
Understanding DNA Organization in the Nucleus By Fluorescence Microscopy and Energy Filtered Transmission Electron Microscopy
}

\author{
D.P. Bazett-Jones
}

Programme in Cell Biology, The Hospital For Sick Children, 555 University Avenue, Toronto, ON M5G 1X8, Canada

Molecular imaging techniques provide structural information, often down to the sub-nanometer level of spatial resolution, complementing x-ray crystallography and magnetic resonance spectroscopy. Molecular imaging offers some advantages; for example, complexes that have not been crystallized can be examined, and very small amounts of purified material are often sufficient. Energy filtered transmission electron microscopy is a very sensitive analytical electron microscopic technique that can generate high resolution distribution maps and quantification of particular chemical elements. The technique has allowed us to map DNA or RNA in nucleoprotein complexes and to determine stoichiometric relationships of protein and nucleic acid in these complexes. One of these complexes is condensin, a five-subunit protein complex essential for mitotic chromosome condensation from yeast to humans, introduces positive supercoils into DNA in an ATP-dependent manner in vitro [1]. We report here the direct visualization of this supercoiling reaction by electron spectroscopic imaging. In the presence of ATP, a single condensin complex is capable of introducing $\geq 2$ compensatory supercoils into the protein-free region of a closed circular DNA. Within the condensin-bound region, $\sim 190 \mathrm{bp}$ of DNA is organized into a compact structure with two distinct domains, indicative of the formation of two oriented gyres. The current results suggest that the action of condensin is more dynamic and more efficient than that postulated before, providing fundamental insight into the energy-dependent mechanism of higher-order chromatin folding.

Regulated transcription requires accurate temporal assembly of complex machines involving scores of components onto chromatin templates of specific genes at precisely the right time. Microscopical evidence indicates that the nucleus is organized into compartments and sub-domains, which could provide local environments that, at least fine tune transcription from a particular gene promoter, if not dictate whether transcription from that promoter will proceed at all. More fundamental questions, however, are: How do proteins move throughout the nucleus? What is the physical basis for nuclear sub-domains? How are these sub-domains assembled and disassembled, and maintained at the proper size, volume and location relative to chromosome territories within the nucleus?

Energy filtered transmission electron microscopy, in combination with fluorescence and live cell microscopy, is well suited to such in situ studies of nuclear domains because elemental mapping can be used to delineate biochemical features. Two subnuclear domains are shown in the figures, identified as local accumulations of a protein called promyelocytic leukemia protein (PML) and glucocorticoid receptor (GR). By comparisons of nitrogen and phosphorus maps, it is apparent that the PML has a protein-based core (nitrogen-rich, phosphorus-poor), whereas the core of the GR body contains both a fibrous protein-based structure and nucleic acid accumulations. A combination of biochemical and microscopical techniques should lead to an understanding of the structure of these domains. 


\section{References:}

[1] K.Kimura et al., Cell 98 (1999) 239.

[2] This research was supported by operating grants from the Cancer Research Society, Inc., the Canadian Institutes of Health Research and the Natural Sciences and Engineering Research Council. The author holds a Canada Research Chair in Molecular and Cellular Imaging.
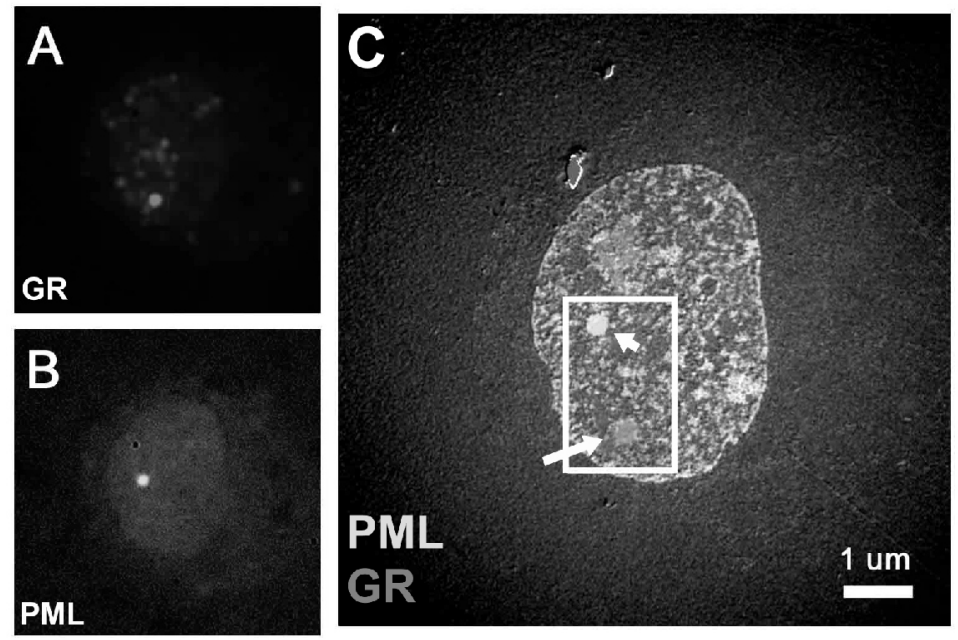

FIG. 1 Fluorescence image of thin section of cell labelled with labelled antibodies against PML protein and GR protein (A, B). The fluorescence image was superimposed on an energy filtered (C) (150 eV energy loss) image of the same section to identify the PML (short arrow) and GR (long arrow) accumulations in the nucleus.
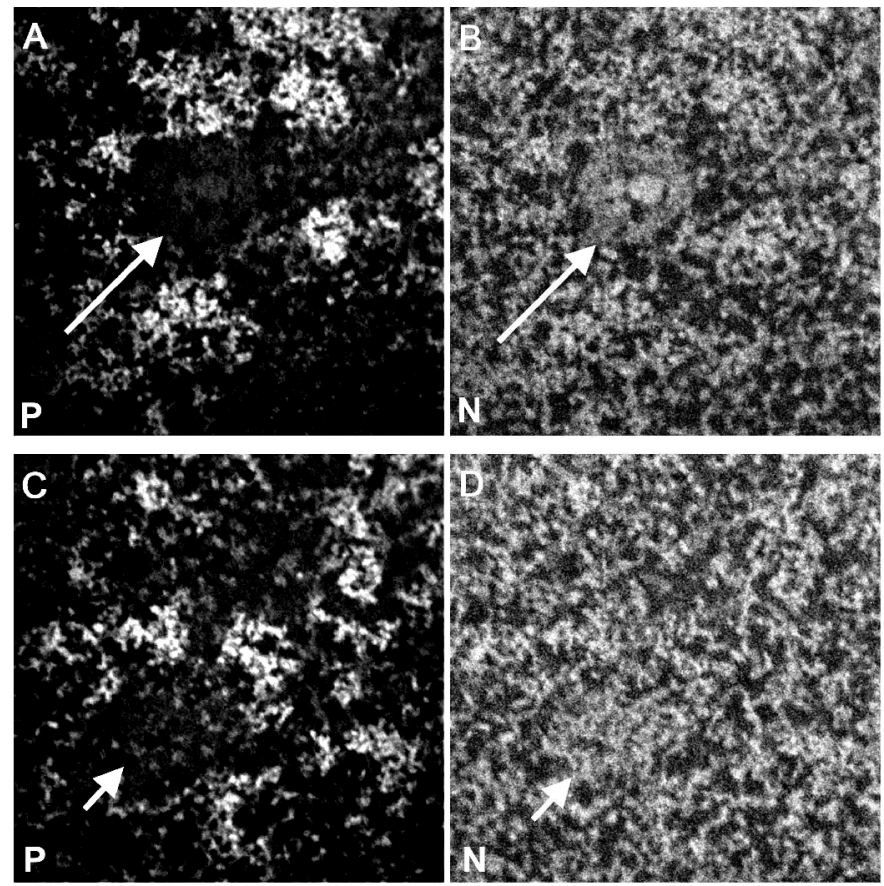

FIG. 2 Energy filtered images at higher magnification of the PML (A, B) and the GR (C, D) accumulations imaged in FIG. 1. Net phosphorus maps $(\mathrm{A}, \mathrm{C})$ and net $\mathrm{N}$ maps $(\mathrm{B}$, D) can be compared to identify protein-based and nucleic acid-based structures. Both nuclear bodies are composed of a protein-based core (arrows) and are surrounded by blocks of chromatin. 